\title{
Modulation of Erythrocyte Plasma Membrane Redox System Activity by Curcumin
}

\author{
Prabhakar Singh, ${ }^{1}$ Rajesh Kumar Kesharwani, ${ }^{2}$ Krishna Misra, ${ }^{2}$ and Syed Ibrahim Rizvi ${ }^{1}$ \\ ${ }^{1}$ Department of Biochemistry, University of Allahabad, Allahabad 211002, India \\ ${ }^{2}$ Division of Applied Science \& Indo-Russian Center For Biotechnology (IRCB), Indian Institute of Information Technology, \\ Allahabad 211012, India \\ Correspondence should be addressed to Syed Ibrahim Rizvi; sirizvi@gmail.com
}

Received 17 October 2015; Revised 30 December 2015; Accepted 31 December 2015

Academic Editor: David L. Daleke

\begin{abstract}
Copyright (c) 2016 Prabhakar Singh et al. This is an open access article distributed under the Creative Commons Attribution License, which permits unrestricted use, distribution, and reproduction in any medium, provided the original work is properly cited.
\end{abstract}

\begin{abstract}
Plasma membrane redox system (PMRS) is an electron transport chain system ubiquitously present throughout all cell types. It transfers electron from intracellular substrates to extracellular acceptors for regulation of redox status. Curcumin, isolated from Curcuma longa, has modulatory effects on cellular physiology due to its membrane interaction ability and antioxidant potential. The present study investigates the effect of curcumin on PMRS activity of erythrocytes isolated from Wistar rats in vitro and in vivo and validated through an in silico docking simulation study using Molegro Virtual Docker (MVD). Effects of curcumin were also evaluated on level of glutathione (GSH) and the oxidant potential of plasma measured in terms of plasma ferric equivalent oxidative potentials (PFEOP). Results show that curcumin significantly $(p<0.01)$ downregulated the PMRS activity in a dose-dependent manner. Molecular docking results suggest that curcumin interacts with amino acids at the active site cavity of cytochrome $b_{5}$ reductase, a key constituent of PMRS. Curcumin also increased the GSH level in erythrocytes and plasma while simultaneously decreasing the oxidant potential (PFEOP) of plasma. Altered PMRS activity and redox status are associated with the pathophysiology of several health complications including aging and diabetes; hence, the above finding may explain part of the role of curcumin in health beneficial effects.
\end{abstract}

\section{Introduction}

Plasma membrane redox system (PMRS) is an electron transport chain system ubiquitously present in all cell types that transfers electrons from intracellular substrates to extracellular acceptors maintaining redox homeostasis for a successful cell physiology [1]. PMRS has been suggested to play a vital role in reducing oxidative stress; this property has been hypothesized to control the rate of aging, lifespan, and many pathological conditions associated with increased oxidative stress $[2,3]$. Proposed functions of PMRS include maintenance of redox state in proteins, stimulation of cell growth, reduction of lipid hydroperoxides, recycling of $\alpha$-tocopherol, reduction of ferric ion prior to iron uptake by a transferringindependent pathway, and the maintenance of the extracellular concentration of ascorbic acid [2-6]. In addition, PMRS also regulates physiological functions like cell metabolism, cellular growth, activity of ion channels, and cell death against changes in redox potential $[7,8]$. PMRS activity also plays an important role affecting recycling of extracellular ascorbic acid, thus preventing its depletion [9]. PMRS donates electrons to extracellular ascorbate free radical (AFR) derived from intracellular redox molecules like glutathione (GSH), Lascorbic acid, nicotinamide adenine dinucleotide (NADH), and other reduced equivalents $[9,10]$.

Cellular physiology is modulated by oxidative stress mediated changes in redox status specially reduced GSH [11]. GSH is a hydrophilic antioxidant with nucleophilic thiol group and has been reported to participate in regulation of gene expression, protein synthesis, cell proliferation, signal transduction, cytokine production, apoptosis, immune response, and protein glutathionylation [11]. GSH protects the cell and biomolecules against oxidative injury, thus providing a powerful antioxidant defense mechanism against reactive 
oxygen species (ROS) and reactive nitrogen species (RNS) [12-14].

Curcumin ((1E,6E)-1,7-bis(4-hydroxy-3-methoxyphenyl)1,6-heptadiene-3,5-dione), a biphenolic molecule isolated from turmeric (Curcuma longa) has pleotropic health protective effects including antioxidation, anti-inflammation, cardiovascular protection, and anticancerous and antiangiogenic properties $[15,16]$. The broad range of curcumin's biological properties is due to its ability to bind to over 100 identified different molecular targets [17]. Curcumin modulates cellular activity through interacting with membranebound proteins and modulating signaling cascade activity by altering the fluidity of the membrane bilayer [17]. The role of curcumin in regulating redox status is complicated and is not still fully understood. The present study was conducted to investigate the in vitro dose-dependent effect of curcumin on PMRS activity of erythrocytes and validated through in vivo studies on Wistar rats. We also report the in silico docking of curcumin with cytochrome $b_{5}$ reductase comparing MoleDock and $\mathrm{H}$-bond score with natural ligand $\beta$-NADH. The effects of curcumin were also evaluated on redox status in plasma as well as erythrocytes in terms of GSH concentration and plasma ferric equivalent oxidative potentials (PFEOP).

\section{Material and Methods}

2.1. Chemicals and Instrument. Curcumin was purchased from Bio Basic Inc., Ontario, Canada (cat. number \#CB0346). DMPD ( $N, N$-dimethyl- $p$-phenylenediamine dihydrochloride) was purchased from Sigma Aldrich, India. Other chemicals of highest purity were purchased from Merck, India, and HIMEDIA Labs, India. Digital pH meter Cyber scan PH 500 manufactured by Merck was used to measure the $\mathrm{pH}$ of solutions. Spectrophotometric measurements were performed on UV-VIS Spectrophotometer (Shimadzu-UV1800, Japan).

\subsection{Experimental Study}

2.2.1. Animals. Male albino rats (Wistar strain) weighing between 150 and $200 \mathrm{~g}$ were purchased from CDRI, Lucknow, India. Animals were housed in polypropylene cages (6 rats per cage) at $24 \pm 2^{\circ} \mathrm{C}$ under $12 \mathrm{~h}$ light: $12 \mathrm{~h}$ dark cycles. Animals were fed with standard pellet diet obtained from Dayal Industries Limited, Lucknow, India, and had free access to drinking water. The protocol of the study was in conformity with the guidelines of the Institutional Ethical Committee, University of Allahabad. Twenty-four rats were randomly divided into four groups (six rats/group): Group (I): control, receiving no treatment/supplementation; Group (II): experimental control, rats that were supplemented with $0.9 \%$ $\mathrm{NaCl}$ solution through oral route; Group (III): curcumin treated group (340 mg/kg b.w., saline); Group (IV): curcumin treated group (170 mg/kg b.w., saline). Oral supplementation of curcumin and sacrifices of rats were performed according to the method of Singh and Rizvi [18]. was centrifuged at $800 \times \mathrm{g}$ at $4^{\circ} \mathrm{C}$ for $10 \mathrm{~min}$. Fresh plasma was used for experimental analyses. After the removal of plasma, buffy coat, and upper $15 \%$ of the packed cells, remaining PRBCs were washed twice with $10 \mathrm{mM}$ phosphate buffer saline $\mathrm{pH}$ 7.4. In vitro effects of curcumin were observed by incubating washed erythrocytes with curcumin $\left(10^{-5} \mathrm{M}\right.$ to $10^{-8} \mathrm{M}$ ) for $30 \mathrm{~min}$ at $37^{\circ} \mathrm{C}$ as described previously [19].

2.2.3. Measurement of PMRS Activity. The PMRS activity was measured following the method of Avron and Shavit [20]. Briefly $0.2 \mathrm{~mL}$ of PRBCs was suspended in phosphate buffer saline (PBS) containing $5 \mathrm{mM}$ glucose and $1 \mathrm{mM}$ potassium ferricyanide (fresh) to a final volume of $2.0 \mathrm{~mL}$. The suspensions were incubated for $30 \mathrm{~min}$ at $37^{\circ} \mathrm{C}$ and then centrifuged at $1000 \times \mathrm{g}$ at $4^{\circ} \mathrm{C}$ for $5 \mathrm{~min}$. The collected supernatant was assayed for ferrocyanide content using 4,7-diphenyl-1,10phenanthroline disulfonic acid disodium salt and measuring absorption at $535 \mathrm{~nm}$. The activity of PMRS was calculated by using extinction coefficient, $\mathscr{E}=20,500 \mathrm{M}^{-1} \mathrm{~cm}^{-1}$. PMRS activity was expressed in $\mu \mathrm{mol}$ ferrocyanide $/ \mathrm{mL}$ $\mathrm{PRBC} / 30$ min.

2.2.4. Measurement of Glutathione. Plasma or erythrocyte GSH was measured according to the method of Beutler [21]. The method is based on the ability of the $-\mathrm{SH}$ group of GSH to reduce 5, $5^{\prime}$-dithiobis,2-nitrobenzoic acid (DTNB) into a yellow colored anionic product whose absorbance was measured at $412 \mathrm{~nm}$. An aqueous solution of known reduced glutathione concentration was used for standard calibration. Concentration of GSH was determined by using standard plot. Concentration of GSH is expressed in $\mu \mathrm{g} / \mathrm{mL}$ plasma and $\mathrm{mg} / \mathrm{mL}$ PRBCs.

2.2.5. Oxidant Potential of Plasma. The plasma oxidant capacity in terms of plasma ferric equivalent oxidative potential (PFEOP) was measured according to method of Mehdi and Rizvi [22]. Briefly $100 \mathrm{mM}$ stock solution of DMPD was prepared by dissolving $209 \mathrm{mg}$ of DMPD in $10 \mathrm{~mL}$ of distilled water. $100 \mathrm{mM}$ acetate buffer ( $\mathrm{pH}$ 5.25) was prepared by mixing of $100 \mathrm{mM}$ sodium acetate $(75.98 \mathrm{~mL})$ and $100 \mathrm{mM}$ acetic acid $(24.02 \mathrm{~mL})$ to bring the $\mathrm{pH} 5.25$. Now $1 \mathrm{~mL}$ of DMPD stock solution was added to $100 \mathrm{~mL}$ acetate buffer (0.1 M, pH 5.25) to bring DMPD concentration to $1 \mathrm{mM}$. A standard curve of ferric chloride was prepared by taking $0.020-0.20 \mathrm{mM}$ ferric chloride as final concentration in $2 \mathrm{~mL}$ solutions $(1.9 \mathrm{~mL}$ DMPD solution with $100 \mu \mathrm{L}$ ferric chloride solutions accordingly). Final solutions were kept for $15 \mathrm{~min}-$ utes and absorbance was taken at $505 \mathrm{~nm}$ against a DMPD blank. $100 \mu \mathrm{L}$ (4 times) of diluted plasma was added to the $1.90 \mathrm{~mL}$ of DMPD working solution (1 $\mathrm{mM}$ in acetate buffer). The whole content was mixed well and kept for incubation for 10 minutes at room temperature and then centrifuged for 5 minutes at $6000 \times \mathrm{g}$ at $4^{\circ} \mathrm{C}$. Absorbance of supernatant was taken at $505 \mathrm{~nm}$ against DMPD solution devoid of plasma as blank. The finding absorbance was compared to ferric iron standard curve for final concentrations. Plasma oxidant capacity is reported as $\mathrm{mM}$ ferric equivalent/L of plasma. 


\subsection{Computational Study}

2.3.1. Protein and Ligands Structure Preparation. Human $\mathrm{NADH}$-cytochrome $b_{5}$ reductase with 3-dimensional structure was downloaded from the protein data bank (pdb id $=1 \mathrm{umk}$, resolution $=1.75 \AA)[23,24]$. Input file of protein was prepared by using Molegro Virtual Docker (MVD). The missing hydrogen atom and bond order information were assigned. The active site cavity of target protein was selected based on Kesharwani et al. [25]. The structure of curcumin and other selected ligands were downloaded from pubchem database and prepared by using MVD.

2.3.2. Docking Simulation. Docking simulation study was performed by Molegro Virtual Docker (MVD), an automated docking software on HP Z800 workstation. The simulation results returned five different poses with their MoleDock and H-bond score [26].

2.4. Statistical Analysis. Statistical analysis was performed by Graph Pad Prism 5 version 5.01 (Graphpad Software Inc., San Diego, California, USA). One way analysis of variance (ANOVA) was done to assess relationships between parameters followed by Bonferroni's multiple comparison test for comparisons between the various treated groups. All the values with $p<0.05$ were considered as statistically significant.

\section{Results}

Figure 1 shows that in vitro curcumin $\left(10^{-5} \mathrm{M}\right.$ to $\left.10^{-8} \mathrm{M}\right)$ triggered concentration dependent downregulation of PMRS activity in erythrocytes obtained from Wistar rats. Maximum downregulation of PMRS activity was observed at $10^{-5} \mathrm{M}$ ( $p<0.001$ ) of curcumin; decreasing effect was observed on lowering the concentration of curcumin till $10^{-8} \mathrm{M}$. In vivo oral supplementation of curcumin (340 and $170 \mathrm{mg} / \mathrm{kg}$ b.w.) to rats significantly ( $p<0.001)$ reduced the PMRS activity of erythrocytes as compared with control and experimental control rats (Figure 2). A less pronounced effect was observed at lower concentrations of curcumin; the effect was similar to in vitro observations. Figure 3 shows a proposed action mechanism of curcumin for PMRS activity.

The secondary structure (cartoon) representation of active site of human NADH-cytochrome $b_{5}$ reductase (1 umk.pdb) together with docked conformation of ligands is shown in Figure 4. Molecular docking simulation results depicted in Figure 5(a) and Table 1 show the active site residues of catalytic unit of human $\mathrm{NADH}$-cytochrome $b_{5}$ reductase (1 umk.pdb). Arg91, Tyr93, Ile109, Lys110, Tyr112, Phe113, Thr116, His117, Phe120, Gly123, Gly124, Lys125, Met126, Ser127, Thr181, and Gln210 were actively involved in hydrogen bonding and hydrophobic interaction with $\beta$ NADH. Figure 5(b) shows that curcumin interacted actively with His77, Pro92, Tyr93, Thr94, Val108, Ile109, Lys110, Gly179, Thr181, Gly182, Thr184, Pro185, Cys273, and Pro275 amino acids at the catalytic center. Five and two hydrogen bonds were formed with amino acids present at the active site by $\beta$-NADH and curcumin, respectively. Table 2 shows that

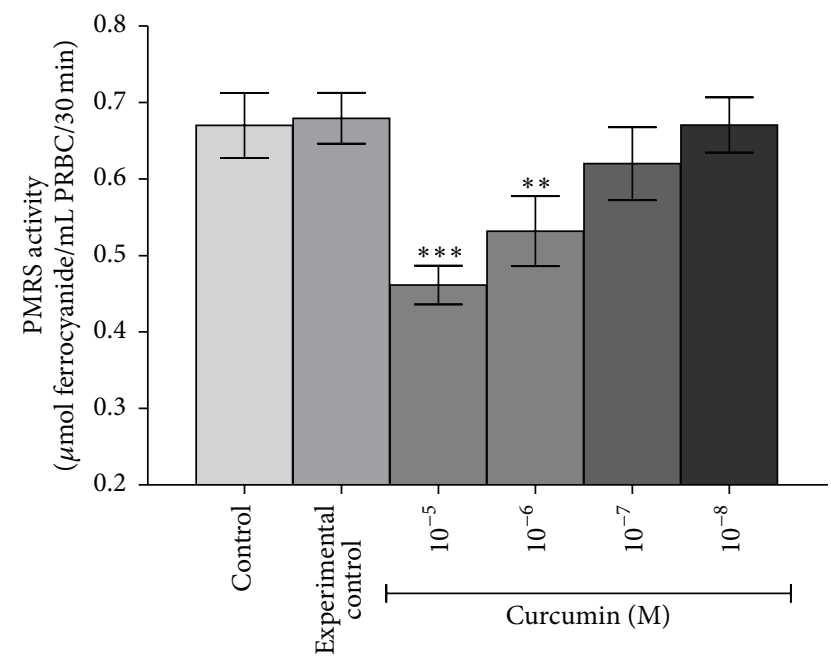

FIGURE 1: In vitro effects of curcumin $\left(10^{-5} \mathrm{M}\right.$ to $\left.10^{-8} \mathrm{M}\right)$ on Wistar rat erythrocytes PMRS activity: PMRS activity expressed in terms of $\mu \mathrm{mol}$ ferrocyanide/mL PRBC/30 min. Values represent mean $\pm \mathrm{SD}$. Control represents erythrocytes receiving no treatment; Experimental control: erythrocytes were incubated with solvent, that is, $0.1 \%$ DMSO.

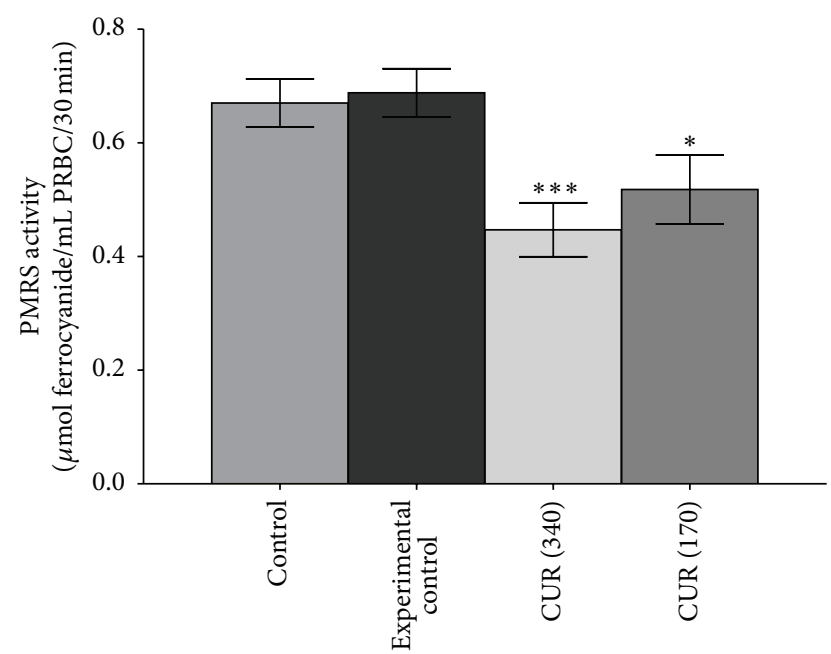

FIGURE 2: In vivo effect of curcumin $(340 \mathrm{mg} / \mathrm{kg}$ b.w. as CUR 340 and $170 \mathrm{mg} / \mathrm{kg} \mathrm{b.w.} \mathrm{oral} \mathrm{CUR} \mathrm{170)} \mathrm{on} \mathrm{healthy} \mathrm{rat} \mathrm{erythrocytes}$ membrane PMRS activity. PMRS activity expressed in terms of $\mu$ mol ferrocyanide/mL PRBC/30 min. Values represent mean \pm SD. Control represents rats receiving no treatment/supplementation; experimental control: rats were supplemented with $0.9 \% \mathrm{NaCl}$ solution through oral route.

the MoleDock score and H-binding energy of curcumin were relatively lower when compared with natural ligand of $\mathrm{NADH}$-cytochrome $b_{5}$ reductase $(\beta$-NADH).

Figure 6 shows that, in vitro, incubation of curcumin $\left(10^{-5} \mathrm{M}\right.$ to $\left.10^{-8} \mathrm{M}\right)$ with rat erythrocytes significantly $(p<$ 0.001 ) increased the GSH concentration; the maximum effect was observed at $10^{-5} \mathrm{M}$ of curcumin; lower concentrations elicited lesser response. In vivo, curcumin (340 


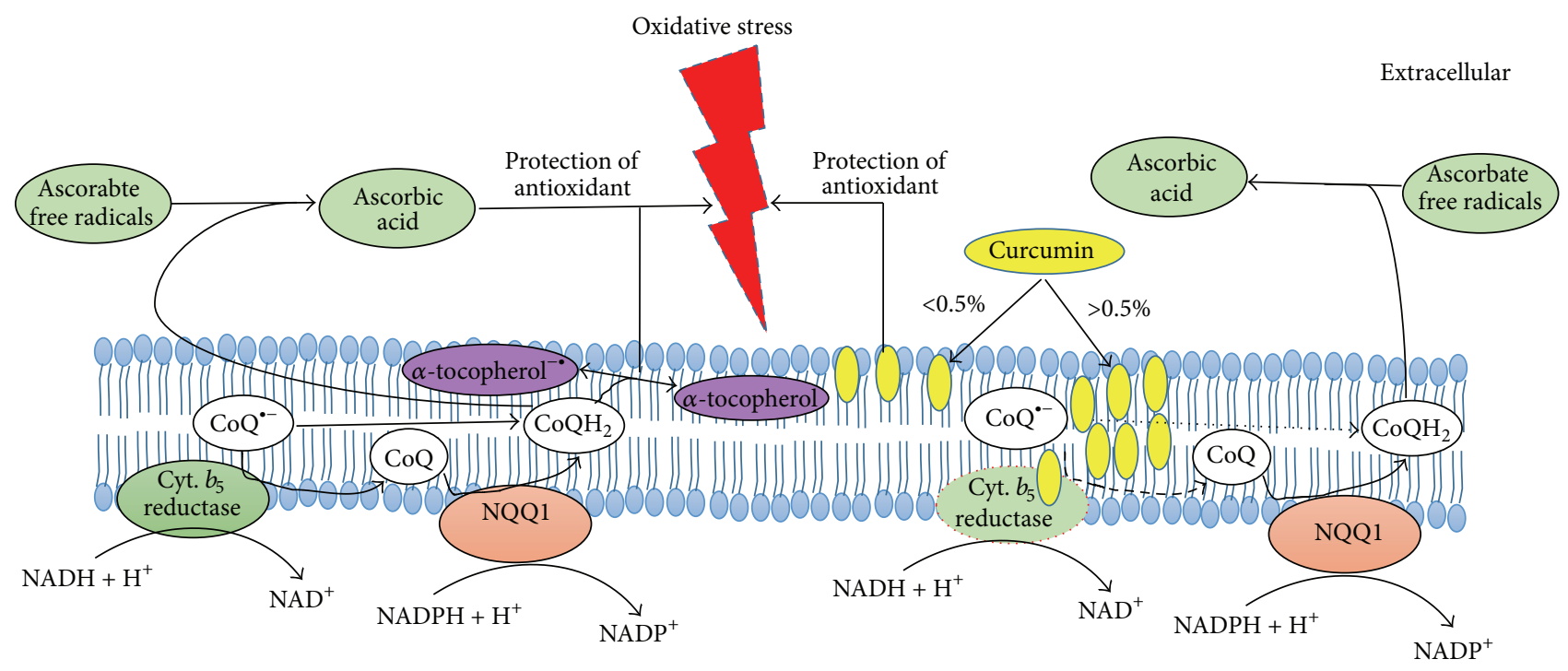

Absence of curcumin

Presence of curcumin

Intracellular

FIGURE 3: Proposed action mechanism of curcumin: dotted outline of PMRS component and arrow indicates the active targets of curcumin towards downregulation of the PMRS activity.

TABLE 1: Amino acids showing hydrogen bond (italic) and hydrophobic interaction.

\begin{tabular}{ll}
\hline S. number & Amino acids interaction \\
\hline$\beta$-NADH & Arg91, Tyr93, Ile109, Lys110, Tyr112, Phe113, Thr116, His117, Phe120, Gly123, Gly124, Lys125, Met126, Ser127, Thr181, \\
& Gln210 \\
Curcumin (CUR) & His77, Pro92, Tyr93, Thr94, Val108, Ile109, Lys110, Gly179, Thr181, Gly182, Thr184, Pro185, Cys273, Pro275 \\
\hline
\end{tabular}

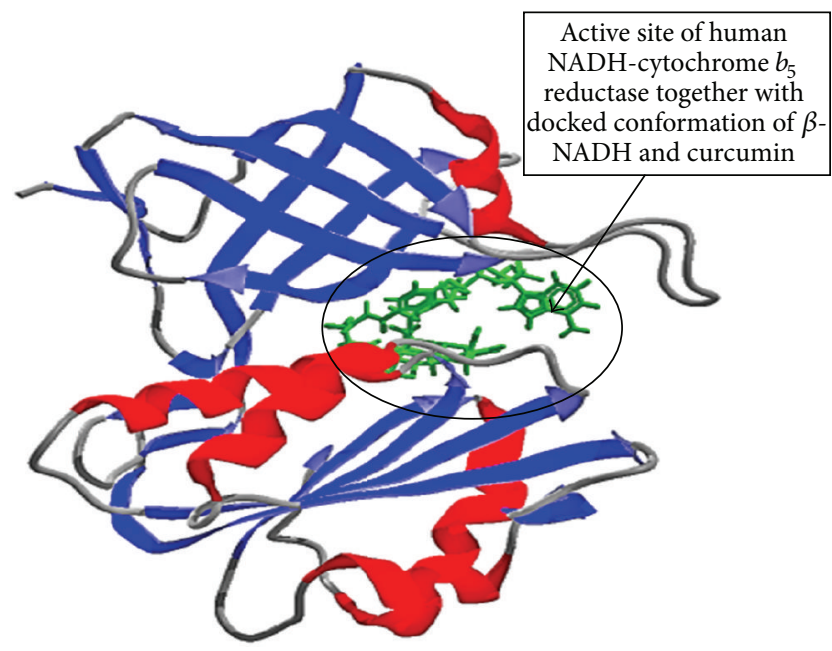

FIGURE 4: Secondary structure (cartoon) representation at the active site of human NADH-cytochrome $b_{5}$ reductase together with docked conformation of ligand $\beta-\mathrm{NADH}$ and curcumin.

and $170 \mathrm{mg} / \mathrm{kg}$ b.w.) supplementation significantly $(p<$ 0.001 ) increased GSH concentration in plasma as well as erythrocytes (Figures $7(\mathrm{a})$ and $7(\mathrm{~b})$ ). Figure 8 shows that in vivo curcumin (340 and $170 \mathrm{mg} / \mathrm{kg}$ b.w.) supplementation significantly $(p<0.001)$ reduced the oxidant potential of plasma (PFEOP).

\section{Discussion}

The erythrocyte PMRS plays an important role in regulating antioxidant status of the plasma during aging and progression of age associated diseases $[27,28]$. Rizvi et al. proposed that long living species have inherent higher PMRS activity in erythrocyte which provides an effective armament to combat oxidative stress [2]. Dietary polyphenols have modulatory effects on PMRS activity which is linked to their cellular internalization [3]. Curcumin has been reported to interact with phospholipid head groups in the membrane bilayer through hydrogen bonds at low concentrations and hydrophobic tail region at high concentrations; these interactions may induce changes in membrane fluidity and associated transport system activity $[29,30]$.

Our results show that curcumin downregulate PMRS activity in healthy erythrocytes in vitro (Figure 1) as well as in vivo (Figure 2 ) in a dose-dependent manner. The observed effect might be due to the biphasic interaction of curcumin 
TABLE 2: Comparative docking simulation result of selected molecule and curcumin with human NADH-cytochrome $b_{5}$ reductase together with FAD, ligand from X-ray crystallized data of protein data bank (1 umk.pdb) using Molegro Virtual Docker (MVD).

\begin{tabular}{lcccc}
\hline S. number & Ligands & MoleDock score & H-bonding energy & Number of H-bonds \\
\hline$(1)$ & $\beta$-NADH & -208.235 & -13.506 & 5 \\
$(2)$ & Curcumin & -141.292 & -5.37453 & 2 \\
\hline
\end{tabular}

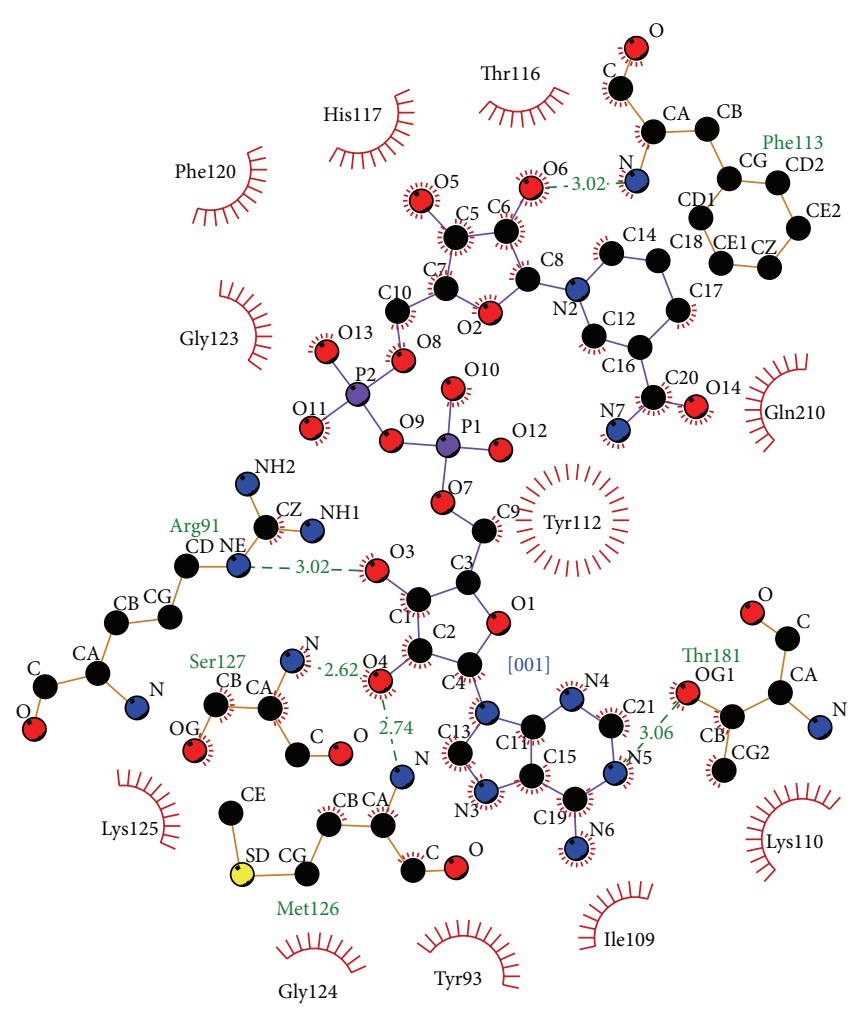

(a)

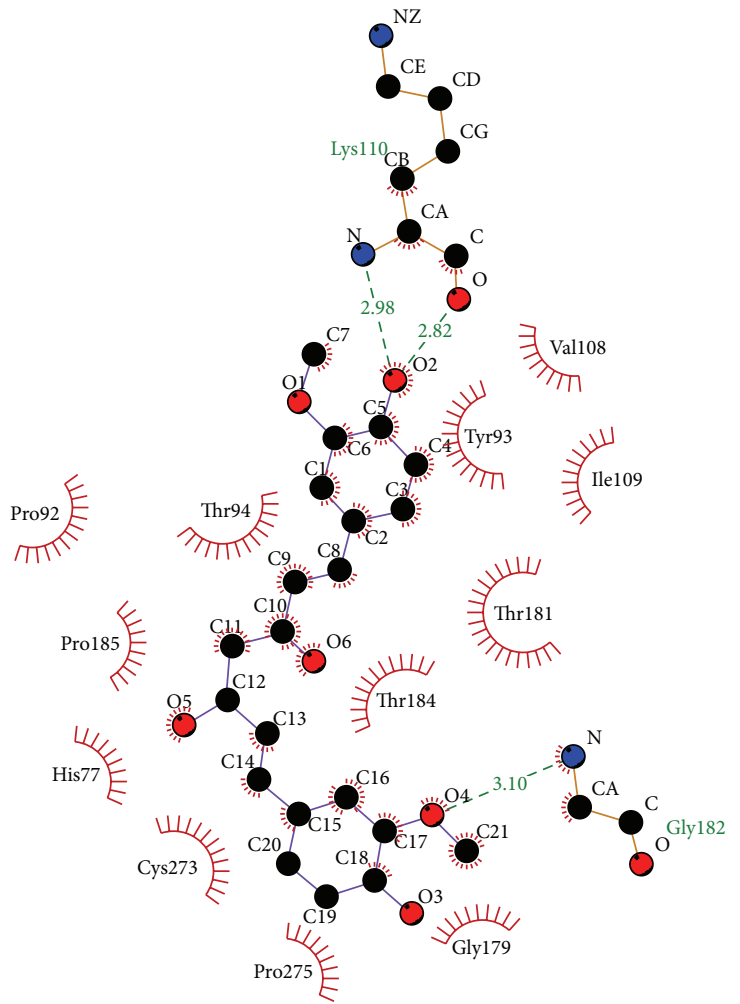

(b)

FIGURE 5: $(\mathrm{a}, \mathrm{b})$ Docked conformation of hydrogen bonding view and hydrophobic interaction of (a) $\beta$-NADH and (b) curcumin with amino acids of human NADH-cytochrome $b_{5}$ reductase protein (PDB: 1 umk.pdb) at the active site cavity (hydrogen bonds as green dashed lines between the atoms involved and hydrophobic contacts as an arc with spokes radiating towards the ligand atoms).

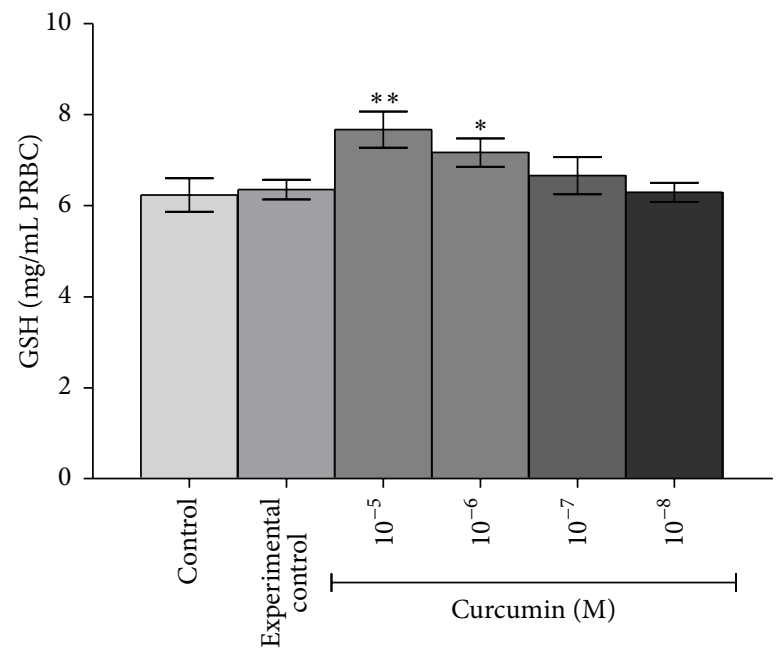

FIGURE 6: In vitro effects of curcumin $\left(10^{-5} \mathrm{M}\right.$ to $\left.10^{-8} \mathrm{M}\right)$ on GSH concentration in Wistar rat RBCs. Concentration of GSH is expressed in $\mathrm{mg} / \mathrm{mL}$ PRBCs. Values represent mean $\pm \mathrm{SD}$. Control represents erythrocytes receiving no treatment; experimental control: erythrocytes were incubated with solvent, that is, $0.1 \%$ DMSO. 


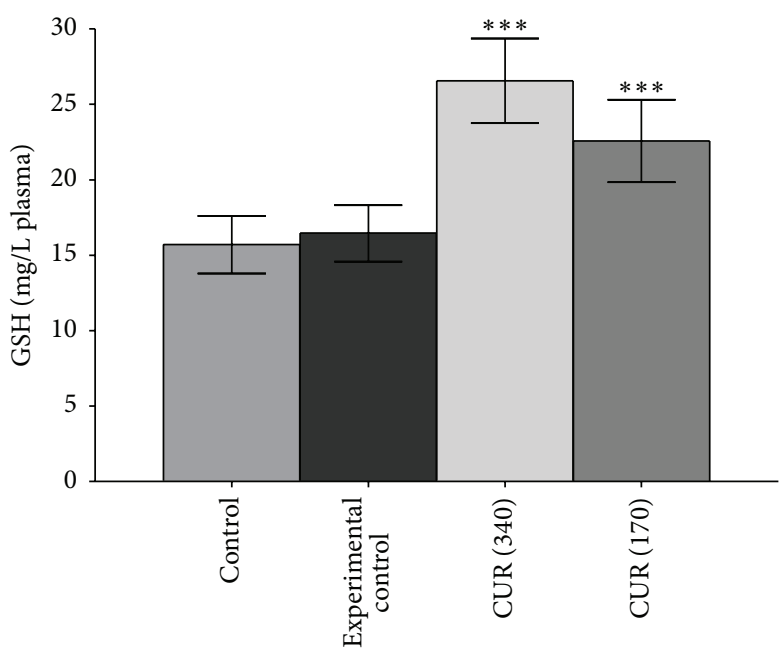

(a)

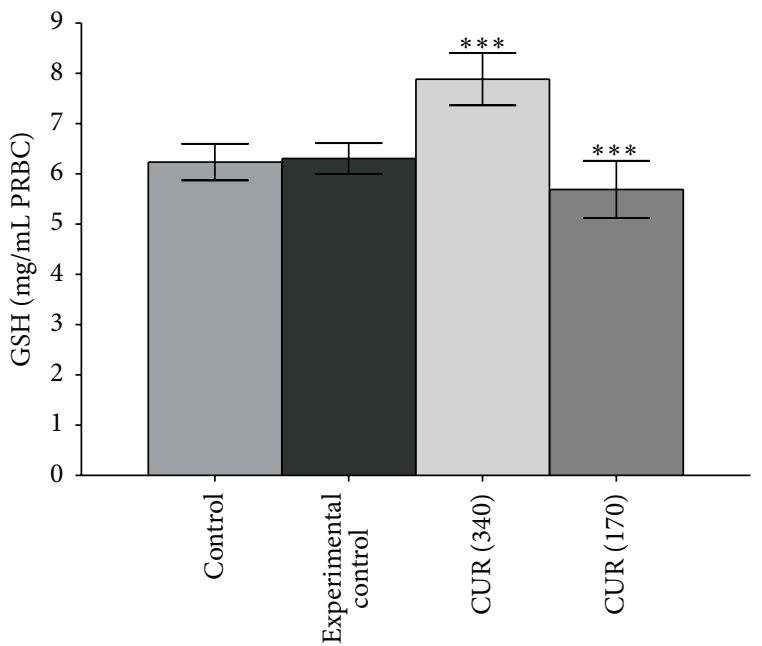

(b)

Figure 7: In vivo effect of curcumin ( $340 \mathrm{mg} / \mathrm{kg}$ b.w. as CUR 340 and $170 \mathrm{mg} / \mathrm{kg}$ b.w. oral CUR 170) on GSH concentration in rats (a) plasma and (b) erythrocytes. Concentration of GSH is expressed in $\mu \mathrm{g} / \mathrm{mL}$ plasma and $\mathrm{mg} / \mathrm{mL}$ PRBCs. Values represent mean \pm SD. Control represents rats receiving no treatment/supplementation; experimental control: rats were supplemented with $0.9 \% \mathrm{NaCl}$ solution through oral route.

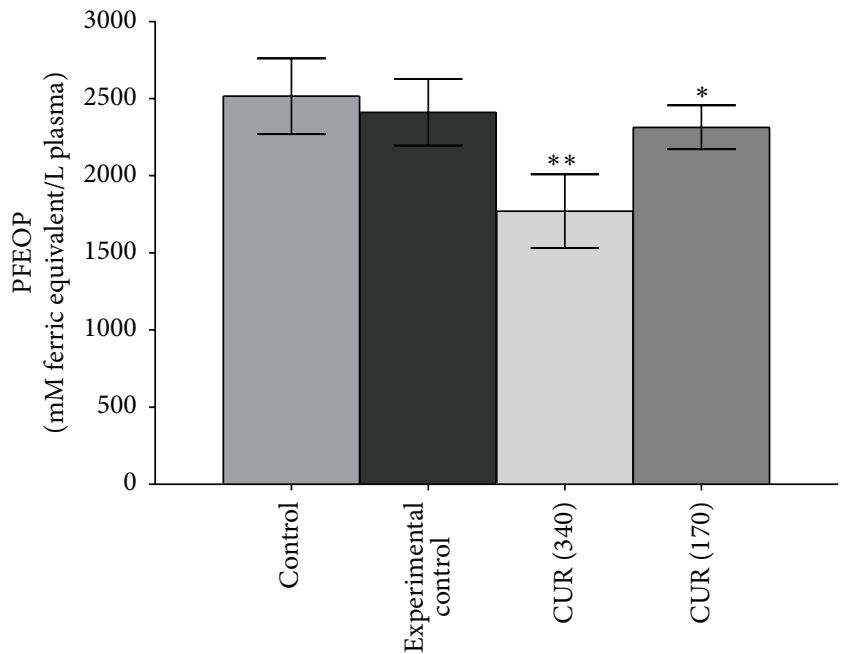

FIGURE 8: In vivo effect of curcumin $(340 \mathrm{mg} / \mathrm{kg}$ b.w. as CUR 340 and $170 \mathrm{mg} / \mathrm{kg}$ b.w. oral CUR 170) on plasma oxidant potential of rat subjected. Plasma oxidant potential is expressed in $\mathrm{mM}$ ferric equivalent $/ \mathrm{L}$ plasma. Values represent mean $\pm \mathrm{SD}$. Control represents rats receiving no treatment/supplementation; experimental control: rats were supplemented with $0.9 \% \mathrm{NaCl}$ solution through oral route.

with membrane bilayer and/or interacting with amino acids at the active site of PMRS. A hypothetical proposed molecular mechanism of curcumin on PMRS activity is depicted in given Figure 3.

PMRS consists of oxidoreductases and electron carrier protein as major entities in which cytochrome $b_{5}$ reductase (EC 1.6.2.2) is a key enzyme. The proposed mechanism elucidates that interaction of curcumin with head groups of membrane can mitigate oxidative stress outside of membrane without influencing PMRS activity. However, interaction at hydrophobic tail region of membrane at higher concentration of curcumin reduces the activity of PMRS.

Figure 4, shows the active site of human NADHcytochrome $b_{5}$ reductase ( 1 umk.pdb). Results of molecular docking simulation suggest that curcumin actively interacted to form hydrogen bond with amino acids: Lys110 and Gly182, and hydrophobic interaction with His77, Pro92, Tyr93, Thr94, Val108, Ile109, Gly179, Thr181, Thr184, Pro185, Cys273, and Pro275 amino acids at the active site cavity. Results show that curcumin has a favorable MoleDock score due to hydrophobic and H-bonding interaction; however, competitive interaction of curcumin with natural ligands $\beta$ $\mathrm{NADH}$ to NADH-cytochrome $b_{5}$ reductase require higher concentration of curcumin to downregulate the NADHcytochrome $b_{5}$ reductase activity.

Mitochondria-deficient cells ( $\rho^{0}$ cells) survived through maintaining ratio of NAD/NADH and reduced coenzyme Q by upregulating the PMRS activity [31]. Intracellular redox molecule like GSH has been reported to interact and donate protons to the NADH-cytochrome $b_{5}$ reductase [32]. Increased PMRS activity has been reported to associate with depletion of intracellular GSH concentration [33]. It has been reported that "redox buffering" capacity of cellular system is substantiated primarily by GSH, an indicator of the redox status of the cell [34]. Cells with low levels of GSH have been shown to be more susceptible towards irradiation and stress [35].

Here, we found that, in vitro, curcumin induced the redox status of erythrocytes evidenced as increased GSH concentration (Figure 6). Oral supplementation of curcumin to healthy rat also induced the GSH concentration in plasma (Figure 7(a)) as well as in erythrocytes (Figure 7(b)). It has been observed previously that oral dosing of curcumin to rats increased the hepatic cells redox status via increasing the activity of $\gamma$-GCS (the rate limiting step in GSH synthesis), 
GPx, and GST [36]. Previously we have also reported that curcumin strongly protects the GSH level in human erythrocytes against oxidative stress [19]. Our observation that curcumin supplementation reduced the PFEOP value of healthy rats indicate a decreased vulnerability of erythrocyte towards oxidative stress and hence reduced PMRS activity.

\section{Conclusion}

Results indicate that curcumin interacts with membrane bilayer to downregulate the plasma redox system activity, the effect being more pronounced at higher concentration $\left(>10^{-6} \mathrm{M}\right)$. In addition, curcumin also stimulates the intracellular GSH while simultaneously decreasing the oxidant capacity of plasma. Impaired redox status and altered PMRS activity are involved in various age associated diseases. The above findings provide evidence of curcumin to be an effective molecule which may be used as a supplement to combat pathologies associated with oxidative stress. Moreover the results may, in part, explain the efficacy of traditional therapeutic strategies based on the use of turmeric in prevention of age-dependent pathologies.

\section{Abbreviations}

PMRS: Plasma membrane redox system

CUR: Curcumin

GSH: Reduced glutathione

PFEOP: Plasma ferric equivalent oxidative potentials.

\section{Conflict of Interests}

The authors declare that there is no conflict of interests regarding this paper.

\section{Acknowledgments}

The work is supported by Council of Scientific and Industrial Research, New Delhi, India, in the form of fellowship to Prabhakar Singh as Senior Research Fellow. Rajesh Kumar Kesharwani acknowledges CLC Bio for providing license to use MVD software. Department of Biochemistry, University of Allahabad, is supported by FIST grant from Department of Science and Technology, Govt. of India.

\section{References}

[1] A. D. N. J. de Grey, "The plasma membrane redox system: a candidate source of aging-related oxidative stress," Age, vol. 27, no. 2, pp. 129-138, 2005.

[2] S. I. Rizvi, D. Kumar, S. Chakravarti, and P. Singh, "Erythrocyte plasma membrane redox system may determine maximum life span," Medical Hypotheses, vol. 76, no. 4, pp. 547-549, 2011.

[3] S. I. Rizvi and N. Srivastava, "Erythrocyte plasma membrane redox system in first degree relatives of type 2 diabetic patients," International Journal of Diabetes Mellitus, vol. 2, no. 2, pp. 119121, 2010.

[4] M. M. Van Duijn, J. Van der Zee, J. VanSteveninck, and P. J. A. Van den Broek, "Ascorbate stimulates ferricyanide reduction in HL-60 cells through a mechanism distinct from the NADH-dependent plasma membrane reductase," The Journal of Biological Chemistry, vol. 273, no. 22, pp. 13415-13420, 1998.

[5] J. M. May, Z.-C. Qu, and J. D. Morrow, "Interaction of ascorbate and $\alpha$-tocopherol in resealed human erythrocyte ghosts: transmembrane electron transfer and protection from lipid peroxidation," The Journal of Biological Chemistry, vol. 271, no. 18, pp. 10577-10582, 1996.

[6] J. M. May, Z.-C. Qu, and C. E. Cobb, "Extracellular reduction of the ascoubate free radical by human erythrocytes," Biochemical and Biophysical Research Communications, vol. 267, no. 1, pp. 118-123, 2000.

[7] P. M. Herst and M. V. Berridge, "Plasma membrane electron transport: a new target for cancer drug development," Current Molecular Medicine, vol. 6, no. 8, pp. 895-904, 2006.

[8] J. D. Ly and A. Lawen, "Transplasma membrane electron transport: enzymes involved and biological function," Redox Report, vol. 8, no. 1, pp. 3-21, 2003.

[9] S. I. Rizvi and K. B. Pandey, "Activation of the erythrocyte plasma membrane redox system by resveratrol: a possible mechanism for antioxidant properties," Pharmacological Reports, vol. 62, no. 4, pp. 726-732, 2010.

[10] E. C. Kennett and P. W. Kuchel, "Redox reactions and electron transfer across the red cell membrane," IUBMB Life, vol. 55, no. 7, pp. 375-385, 2003.

[11] K. B. Pandey and S. I. Rizvi, "Markers of oxidative stress in erythrocytes and plasma during aging in humans," Oxidative Medicine and Cellular Longevity, vol. 3, no. 1, pp. 2-12, 2010.

[12] R. Masella, R. Di Benedetto, R. Varì, C. Filesi, and C. Giovannini, "Novel mechanisms of natural antioxidant compounds in biological systems: involvement of glutathione and glutathionerelated enzymes," Journal of Nutritional Biochemistry, vol. 16, no. 10, pp. 577-586, 2005.

[13] I. Rahman and W. MacNee, "Lung glutathione and oxidative stress: implications in cigarette smoke-induced airway disease," The American Journal of Physiology-Lung Cellular and Molecular Physiology, vol. 277, no. 6, part 1, pp. L1067-L1088, 1999.

[14] F. J. Kelly, "Gluthathione: in defence of the lung," Food and Chemical Toxicology, vol. 37, no. 9-10, pp. 963-966, 1999.

[15] P. Singh and S. I. Rizvi, "Curcumin activates erythrocyte membrane acetylcholinesterase," Letters in Drug Design \& Discovery, vol. 10, no. 6, pp. 550-556, 2013.

[16] P. Singh, K. Pandey, and S. Rizvi, "Curcumin: the yellow molecule with pleiotropic biological effects," Letters in Drug Design \& Discovery, vol. 13, no. 2, pp. 170-177, 2015.

[17] H. I. Ingolfsson, R. E. Koeppe II, and O. S. Andersen, "Curcumin is a modulator of bilayer material properties," Biochemistry, vol. 46, no. 36, pp. 10384-10391, 2007.

[18] P. Singh and S. I. Rizvi, "Role of curcumin in modulating plasma PON1 arylesterase activity and susceptibility to LDL oxidation in oxidatively challenged wistar rats," Letters in Drug Design \& Discovery, vol. 12, no. 4, pp. 319-323, 2015.

[19] P. Singh and S. I. Rizvi, "Anti-oxidative effect of curcumin against tert-butylhydroperoxide induced oxidative stress in human erythrocytes," The Natural Products Journal, vol. 2, no. 1, pp. 69-73, 2012.

[20] M. Avron and N. Shavit, "A sensitive and simple method for determination of ferrocyanide," Analytical Biochemistry, vol. 6, no. 6 , pp. 549-554, 1963.

[21] E. Beutler, Red Cell Metabolism: A Manual of Biochemical Methods, Grune and Stratton, Orlando, Fla, USA, 3rd edition, 1984. 
[22] M. M. Mehdi and S. I. Rizvi, "N,N-dimethyl-p-phenylenediamine dihydrochloride-based method for the measurement of plasma oxidative capacity during human aging," Analytical Biochemistry, vol. 436, no. 2, pp. 165-167, 2013.

[23] S. Bando, T. Takano, T. Yubisui, K. Shirabe, M. Takeshita, and A. Nakagawa, "Structure of human erythrocyte NADHcytochrome b5 reductase," Acta Crystallographica Section D: Biological Crystallography, vol. 60, no. 11, pp. 1929-1934, 2004.

[24] S. Dutta, K. Burkhardt, J. Young et al., "Data deposition and annotation at the worldwide protein data bank," Molecular Biotechnology, vol. 42, no. 1, pp. 1-13, 2009.

[25] R. K. Kesharwani, D. V. Singh, K. Misra, and S. I. Rizvi, "Plant polyphenols as electron donors for erythrocyte plasma membrane redox system: validation through in silico approach," Organic and Medicinal Chemistry Letters, vol. 2, no. 1, pp. 1-12, 2012.

[26] R. Thomsen and M. H. Christensen, "MolDock: a new technique for high-accuracy molecular docking," Journal of Medicinal Chemistry, vol. 49, no. 11, pp. 3315-3321, 2006.

[27] S. I. Rizvi, R. Jha, and P. K. Maurya, "Erythrocyte plasma membrane redox system in human aging," Rejuvenation Research, vol. 9, no. 4, pp. 470-474, 2006.

[28] K. B. Pandey and S. I. Rizvi, "Resveratrol up-regulates the erythrocyte plasma membrane redox system and mitigates oxidation-induced alterations in erythrocytes during aging in humans," Rejuvenation Research, vol. 16, no. 3, pp. 232-240, 2013.

[29] P. Singh, R. K. Kesharwani, K. Misra, and S. I. Rizvi, “The modulation of erythrocyte $\mathrm{Na}^{+} / \mathrm{K}^{+}$-ATPase activity by curcumin," Journal of Advanced Research, vol. 6, no. 6, pp. 1023-1030, 2014.

[30] Y. Sun, C.-C. Lee, W.-C. Hung, F.-Y. Chen, M.-T. Lee, and H. W. Huang, "The bound states of amphipathic drugs in lipid bilayers: study of curcumin," Biophysical Journal, vol. 95, no. 5, pp. 2318-2324, 2008.

[31] J. A. Larm, F. Vaillant, A. W. Linnane, and A. Lawen, "Upregulation of the plasma membrane oxidoreductase as a prerequisite for the viability of human namalwa $\rho^{\circ}$ cells," The Journal of Biological Chemistry, vol. 269, pp. 30097-30100, 1994.

[32] D.-H. Hyun, N. D. Hunt, S. S. Emerson, J. O. Hernandez, M. P. Mattson, and R. de Cabo, "Up-regulation of plasma membraneassociated redox activities in neuronal cells lacking functional mitochondria," Journal of Neurochemistry, vol. 100, no. 5, pp. 1364-1374, 2007.

[33] N. Jaiswal, D. Kumar, and S. I. Rizvi, "Red onion extract (Allium cepa L.) supplementation improves redox balance in oxidatively stressed rats," Food Science and Human Wellness, vol. 2, no. 2, pp. 99-104, 2013.

[34] F. Q. Schafer and G. R. Buettner, "Redox environment of the cell as viewed through the redox state of the glutathione disulfide/ glutathione couple," Free Radical Biology and Medicine, vol. 30, no. 11, pp. 1191-1212, 2001.

[35] A. Meister, "Glutathione metabolism and its selective modification," The Journal of Biological Chemistry, vol. 263, no. 33, pp. 17205-17208, 1988.

[36] J. T. Piper, S. S. Singhal, M. S. Salameh, R. T. Torman, Y. C. Awasthi, and S. Awasthi, "Mechanisms of anticarcinogenic properties of curcumin: the effect of curcumin on glutathione linked detoxification enzymes in rat liver," The International Journal of Biochemistry \& Cell Biology, vol. 30, no. 4, pp. 445456, 1998. 

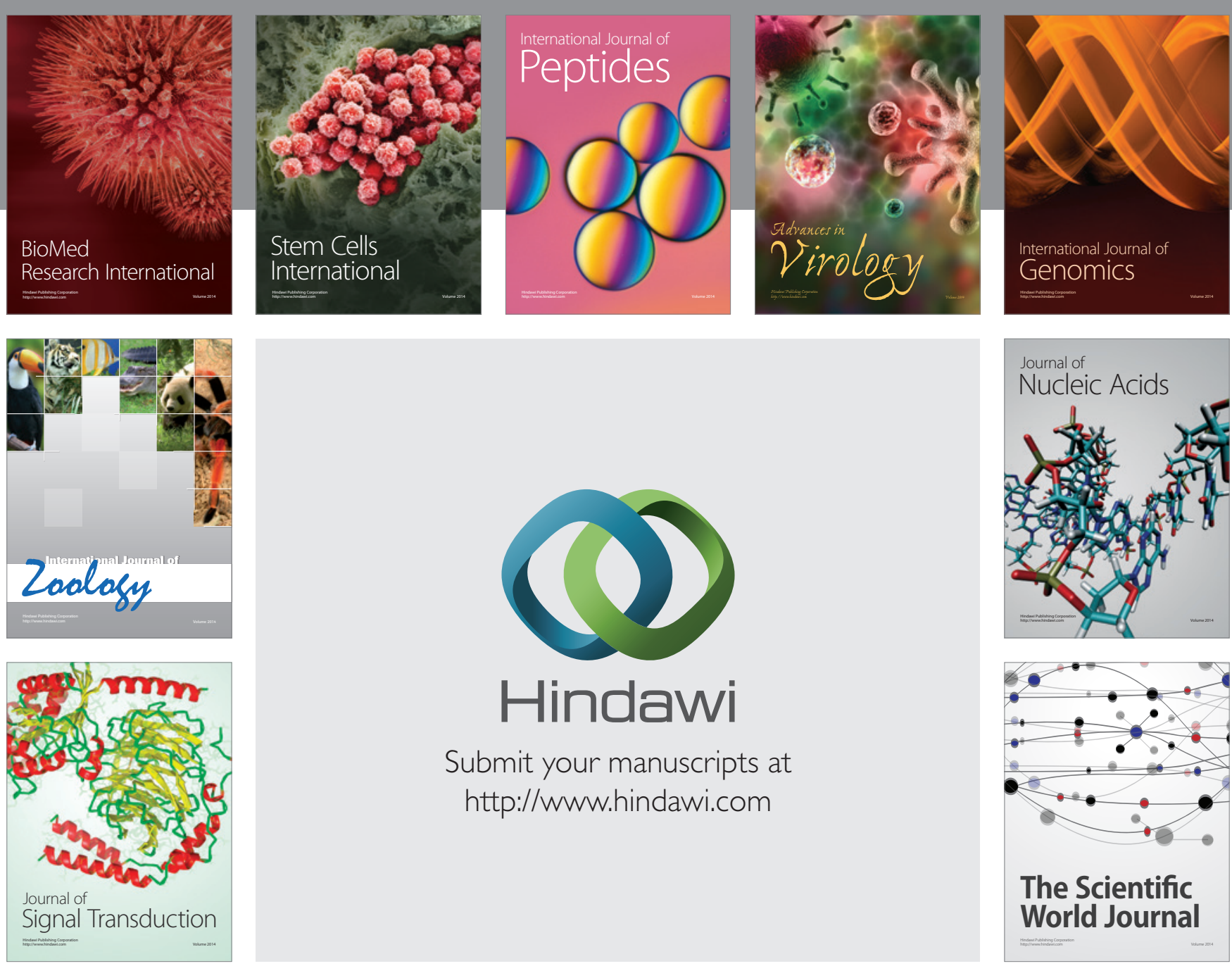

Submit your manuscripts at

http://www.hindawi.com
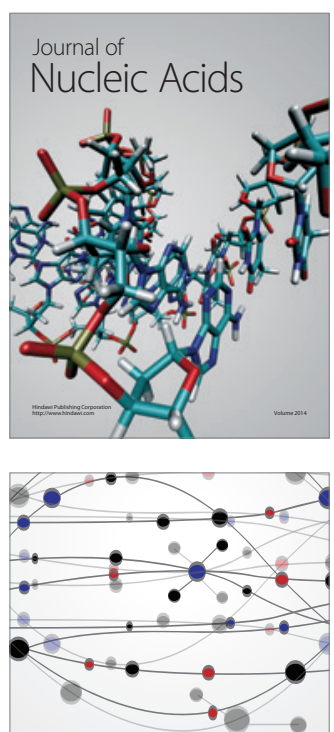

The Scientific World Journal
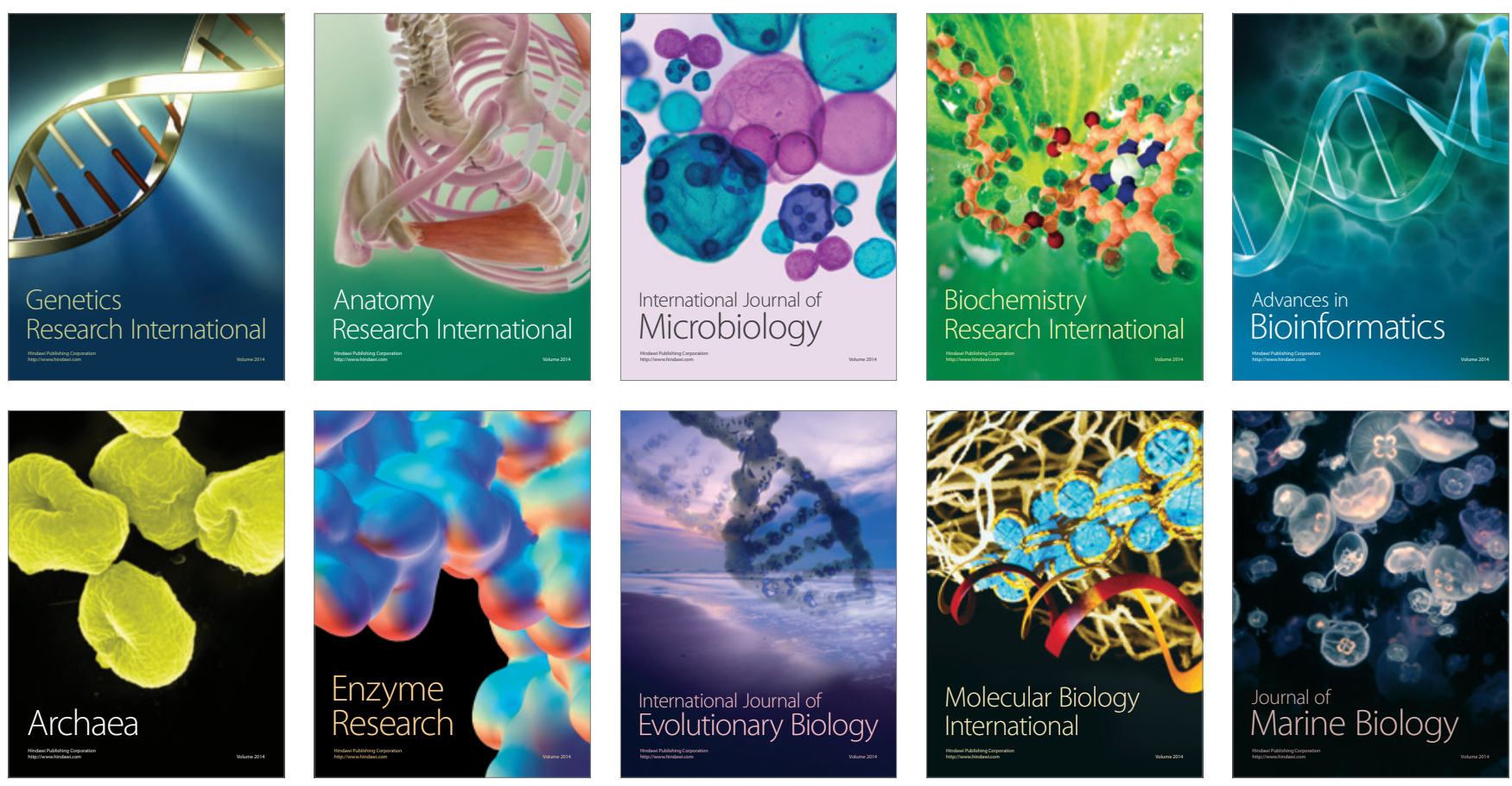